$1-2013$

\title{
Novel use of an ultrasonic bone-cutting device for endoscopic-assisted craniosynostosis surgery
}

Chaichana KL

Johns Hopkins University School of Medicine, Baltimore, MD, USA

Jallo GI

Johns Hopkins University School of Medicine, Baltimore, MD, USA

Dorafshar AH

Johns Hopkins University School of Medicine, Baltimore, MD, USA

Ahn ES

Johns Hopkins University School of Medicine, Baltimore, MD, USA

Follow this and additional works at: http://ecommons.aku.edu/pjns

Part of the Neurology Commons

\section{Recommended Citation}

KL, Chaichana; GI, Jallo; AH, Dorafshar; and ES, Ahn (2013) "Novel use of an ultrasonic bone-cutting device for endoscopic-assisted craniosynostosis surgery," Pakistan Journal of Neurological Sciences (PJNS): Vol. 8 : Iss. 1 , Article 7.

Available at: http://ecommons.aku.edu/pjns/vol8/iss1/7 


\section{NEUROSURGERY}

In this issue, we have selected abstracts of five articles published recently in Neurosurgical journals. The first is a report of the combined of ultrasonic bone-cutting device with endoscopes in craniosynostosis surgery. The report published in Childs Nervous System, carries immense importance since blood loss and operative times are the two major intraoperative concerns for these patients, and the present report suggests improvement in both of these parameters. The next two abstracts concern intracranial pressure monitoring. The first one of these published in Critical Care Medicine, has explored the possibility of predicting changes in intracranial pressures using dynamic data from continuous mean arterial pressure as well as intracranial pressure monitoring. Despite the simplicity of their methods, the researchers report impressive accuracy of their predictions. The study will undoubtedly stimulate many more to emulate these findings. The next is a paper that may turn out to be a landmark publication. This is a multicenter controlled trial published in NEJM which shows that neuro-intensive care traumatic brain injury patients based on continuous monitoring of intracranial pressure is not superior to care based on conventional methods of repeated neuro-imaging and clinical examination. The paper thus questions what had become an accepted standard of neuro-critical care around the world. The next abstract is from Neurosurgeons' favorite journal, the Journal of Neurosurgery. It is first report of utilization of intra-operative MRI and MR Spectroscopy for complete resections of low grade gliomas. The paper suggests that intraoperative MR Spectroscopy is highly sensitive in differentiating residual tumor from nontumoral changes around the resection cavity. The final abstract is a systematic review and meta-analysis of the role of intravenous peri-operative tranexamic acid for reducing blood loss in major spinal surgery. The results are encouraging although the clinical implications are at the moment limited due to a possible predisposition towards venous thromboembolism.

Shahzad Shamim

Department of Surgery (Neurosurgery)

Aga Khan University

\section{Journal: Childs Nerv Syst. 2013 Feb 6. [Epub ahead of print]}

\section{NOVEL USE OF AN ULTRASONIC BONE-CUTTING DEVICE FOR ENDOSCOPIC-ASSISTED CRANIOSYNOSTOSIS SURGERY}

Chaichana KL, Jallo GI, Dorafshar AH, Ahn ES.

Source: Division of Pediatric Neurosurgery, Johns Hopkins University School of Medicine, Baltimore, MD, USA, Kaisorn@jhmi.edu.

Purpose: Endoscopic-assisted craniosynostosis surgery is associated with less blood loss and shorter operative times as compared to open surgery. However, in infants who have low circulating blood volumes, the endoscopic approach is still associated with significant blood loss. A major source of blood loss is the bone that is cut during surgery. We discuss the novel use of an ultrasonic bone-cutting device for craniosynostosis surgery, which decreases bone bleeding. This device, which has primarily only been used for spine and skull base surgery, may help reduce blood loss in these infants.Methods: All patients with single suture craniosynostosis who were operated on with the use of an ultrasonic bone-cutting device were identified. The information retrospectively recorded from patient charts included patient age, suture involved, blood loss, operative times, complications, preoperative hemoglobin, postoperative hemoglobin, length of hospital stay, and follow-up times. Results: Thirteen patients (12 males, 1 female) underwent surgery with an ultrasonic bone-cutting device during the reviewed period. The average age ( \pm standard deviation) of the patients was $11.8( \pm 1.6)$ weeks. Four patients had metopic synostosis and nine patients had sagittal synostosis. The average surgery time was $84( \pm 13) \mathrm{min}$. The median (interquartile range) blood loss was 20 (10-70) cc. No patients required blood transfusions. Three patients had dural tears.Conclusion: We demonstrate the novel use of an ultrasonic bone-cutting device for endoscopicassisted craniosynostosis surgery. This device limited blood loss while maintaining short operative times for infants with low circulating blood volumes. 


\title{
Journal: Crit Care Med. 2013 Feb;41(2):554-64. doi: 10.1097/CCM.0b013e3182742d0a.
}

\author{
NOVEL METHODS TO PREDICT INCREASED INTRACRANIAL \\ PRESSURE DURING INTENSIVE CARE AND LONG TERM \\ NEUROLOGIC OUTCOME AFTER TRAUMATIC BRAIN INJURY: \\ DEVELOPMENT AND VALIDATION IN A MULTICENTER DATASET
}

Güiza F, Depreitere B, Piper I, Van den Berghe G, Meyfroidt G.

Source: Department of Intensive Care Medicine, KU Leuven, Leuven, Belgium. fabian.guiza@med.kuleuven.be

Objective: Intracranial pressure monitoring is standard of care after severe traumatic brain injury. Episodes of increased intracranial pressure are secondary injuries associated with poor outcome. We developed a model to predict increased intracranial pressure episodes 30 mins in advance, by using the dynamic characteristics of continuous intracranial pressure and mean arterial pressure monitoring. In addition, we hypothesized that performance of current models to predict long-term neurologic outcome could be substantially improved by adding dynamic characteristics of continuous intracranial pressure and mean arterial pressure monitoring during the first 24 hrs in the ICU.Design: Prognostic modeling. Noninterventional, observational, retrospective study. Setting and patients: The Brain Monitoring with Information Technology dataset consisted of 264 traumatic brain injury patients admitted to 22 neuroICUs from 11 European countries.Interventions: None. Measurements: Predictive models were built with multivariate logistic regression and Gaussian processes, a machine learning technique. Predictive attributes were Corticosteroid Randomisation After Significant Head Injury-basic and International Mission for Prognosis and Clinical Trial design in TBI-core predictors, together with time-series summary statistics of minute-by-minute mean arterial pressure and intracranial pressure. Main Results: Increased intracranial pressure episodes could be predicted 30 mins ahead with good calibration (Hosmer-Lemeshow $\mathrm{p}$ value 0.12 , calibration slope 1.02, calibration-in-the-large -0.02) and discrimination (area under the receiver operating curve $=0.87$ ) on an external validation dataset. Models for prediction of poor neurologic outcome at six months (Glasgow Outcome Score 1-2) based only on static admission data had 0.72 area under the receiver operating curve; adding dynamic information of intracranial pressure and mean arterial pressure during the first $24 \mathrm{hrs}$ increased performance to 0.90. Similarly, prediction of Glasgow Outcome Score 1-3 was improved from 0.68 to 0.87 when including dynamic information.

Conclusion: The dynamic information in continuous mean arterial pressure and intracranial pressure monitoring allows to accurately predict increased intracranial pressure in the neuro-ICU. Adding information of the first $24 \mathrm{hrs}$ of intracranial pressure and mean arterial pressure monitoring to known baseline risk factors allows very accurate prediction of long-term neurologic outcome at 6 months.

\section{Journal: N Engl J Med. 2012 Dec 27;367(26):2471-81. doi: 10.1056/NEJMoa1207363. Epub 2012 Dec 12.}

\section{A TRIAL OF INTRACRANIAL-PRESSURE MONITORING IN TRAUMATIC BRAIN INJURY}

Chesnut RM, Temkin N, Carney N, Dikmen S, Rondina C, Videtta W, Petroni G, Lujan S, Pridgeon J, Barber J, Machamer J, Chaddock K, Celix JM, Cherner M, Hendrix T.

Source: Department of Neurological Surgery, University of Washington, Harborview Medical Center, Seattle, WA 98104, USA. chesnutr@uw.edu 
Background: Intracranial-pressure monitoring is considered the standard of care for severe traumatic brain injury and is used frequently, but the efficacy of treatment based on monitoring in improving the outcome has not been rigorously assessed.

Methods: We conducted a multicenter, controlled trial in which 324 patients 13 years of age or older who had severe traumatic brain injury and were being treated in intensive care units (ICUs) in Bolivia or Ecuador were randomly assigned to one of two specific protocols: guidelines-based management in which a protocol for monitoring intraparenchymal intracranial pressure was used (pressure-monitoring group) or a protocol in which treatment was based on imaging and clinical examination (imaging-clinical examination group). The primary outcome was a composite of survival time, impaired consciousness, and functional status at 3 months and 6 months and neuropsychological status at 6 months; neuropsychological status was assessed by an examiner who was unaware of protocol assignment. This composite measure was based on performance across 21 measures of functional and cognitive status and calculated as a percentile (with 0 indicating the worst performance, and 100 the best performance).Results: There was no significant between-group difference in the primary outcome, a composite measure based on percentile performance across 21 measures of functional and cognitive status (score, 56 in the pressure-monitoring group vs. 53 in the imaging-clinical examination group; $P=0.49$ ). Six-month mortality was $39 \%$ in the pressuremonitoring group and $41 \%$ in the imaging-clinical examination group $(P=0.60)$. The median length of stay in the ICU was similar in the two groups (12 days in the pressure-monitoring group and 9 days in the imaging-clinical examination group; $P=0.25$ ), although the number of days of brain-specific treatments (e.g., administration of hyperosmolar fluids and the use of hyperventilation) in the ICU was higher in the imagingclinical examination group than in the pressuremonitoring group (4.8 vs. $3.4, \mathrm{P}=0.002$ ). The distribution of serious adverse events was similar in the two groups.

Conclusions: For patients with severe traumatic brain injury, care focused on maintaining monitored intracranial pressure at $20 \mathrm{~mm} \mathrm{Hg}$ or less was not shown to be superior to care based on imaging and clinical examination. (Funded by the National Institutes of Health and others; ClinicalTrials.gov number, NCT01068522.).

\title{
Journal: J Neurosurg. 2013 Feb 22. [Epub ahead of print]
}

\section{INTRAOPERATIVE MAGNETIC RESONANCE SPECTROSCOPY FOR IDENTIFICATION OF RESIDUAL TUMOR DURING LOW GRADE GLIOMA SURGERY}

\author{
Pamir MN, Ozduman K, Yildiz E, Sav A, Dinçer A.
}

Source: Departments of Neurosurgery.

Objective: The authors had previously shown that 3$\mathrm{T}$ intraoperative MRI (ioMRI) detects residual tumor tissue during low-grade glioma and that it helps to increase the extent of resection. In a proportion of their cases, however, the ioMRI disclosed T2-hyperintense areas at the tumor resection border after the initial resection attempt and prompted a differential diagnosis between residual tumor and nontumoral changes. To guide this differential diagnosis the authors used intraoperative long-TE single-voxel proton MR spectroscopy (ioMRS) and tested the correlation of these findings with findings from pathological examination of resected tissue.Methods: Patients who were undergoing surgery for hemispheric or insular WHO Grade II gliomas and were found to have T2 changes around the resection cavity at the initial ioMRI were prospectively examined with ioMRS and biopsies were taken from corresponding localizations. In 14 consecutive patients, the ioMRS diagnosis in 20 voxels of interest was tested against the histopathological diagnosis. Intraoperative diffusion-weighted imaging (ioDWI) was also performed, as a part of the routine imaging, to rule out surgically induced changes, which could also appear as T2 hyperintensity. Results: Presence of tumor was documented in 14 (70\%) of the 20 T2hyperintense areas by histopathological examination. The sensitivity of ioMRS for identifying residual tumor was $85.7 \%$, the specificity was $100 \%$, the positive predictive value was $100 \%$, and the negative predictive value was $75 \%$. The specificity of ioDWI for surgically 
induced changes was high (100\%), but the sensitivity was only $60 \%$.Conclusions: This is the first clinical series to indicate that ioMRS can be used to differentiate residual tumor from nontumoral changes around the resection cavity, with high sensitivity and specificity.

\title{
Journal: PLoS One. 2013;8(2):e55436. doi: 10.1371/journal.pone.0055436. Epub 2013 Feb 12.
}

\section{SYSTEMATIC REVIEW AND META-ANALYSIS OF PERIOPERATIVE INTRAVENOUS TRANEXAMIC ACID USE IN SPINAL SURGERY}

\author{
Yang B, Li H, Wang D, He X, Zhang C, Yang P. \\ Source: Department of Orthopaedic Surgery, The 2nd Affiliated Hospital of Medical College, Xian Jiaotong University, Xian, Shaanxi Province, People's Republic of China.
}

Background: Tranexamic acid (TXA) is well-established as a versatile oral, intramuscular, and intravenous (IV) antifibrinolytic agent. However, the efficacy of IV TXA in reducing perioperative blood transfusion in spinal surgery is poorly documented. Methodology: We conducted a meta-analysis of randomized controlled trials (RCTs) and quasi-randomized (qi-RCTs) trials that included patients for various spinal surgeries, such as adolescent scoliosis surgery administered with perioperative IV TXA according to Cochrane Collaboration guidelines using electronic PubMed, Cochrane Central Register of Controlled Trials, and Embase databases. Additional journal articles and conference proceedings were manually located by two independent researchers. Results: Totally, nine studies were included, with a total sample size of 581 patients. Mean blood loss was decreased in patients treated with perioperative IV TXA by $128.28 \mathrm{ml}$ intraoperatively (ranging from 33.84 to $222.73 \mathrm{ml}$ ), $98.49 \mathrm{ml}$ postoperatively (ranging from
83.22 to $113.77 \mathrm{ml}$ ), and $389.21 \mathrm{ml}$ combined (ranging from 177.83 to $600.60 \mathrm{ml}$ ). The mean volume of transfused packed cells were reduced by $134.55 \mathrm{ml}$ (ranging 51.64 to 217.46$)(95 \% \mathrm{Cl} ; \mathrm{P} ?=? 0.0001)$. Overall, the number of patients treated with TXA who required blood transfusions was lower by $35 \%$ than that of patients treated with the comparator and who required blood transfusions (RR 0.65; 95\% Cl; 0.53 to $0.85 ; \mathrm{P}<0.0001, \mathrm{I}(2) ?=$ ?0\%). A dose-independent beneficial effect of TXA was observed, and confirmed in subgroup and sensitivity analyses. A total of seven studies reported DVT data. The study containing only a single DVT case was not combined.Conclusions: The blood loss was reduced in spinal surgery patients with perioperative IV TXA treatment. Also the percentage of spinal surgery patients who required blood transfusion was significantly decreased. Further evaluation is required to confirm our findings before TXA can be safely used in patients undergoing spine surgery. 